\title{
An IoT Approach for Monitoring UV Disinfection Robots
}

\author{
Conor McGinn ${ }^{1,2}$ and Eamonn Bourke ${ }^{2}$ and Michael F. Cullinan ${ }^{1,2}$
}

\begin{abstract}
The use of ultraviolet germicidal irradiation (UVGI) technology as a means of disinfecting hospitals and other frontline settings has increased significantly in the wake of the COVID-19 pandemic. Although the science of UVGI is well established, it can be difficult to determine in practice if sufficient levels of UVC has been irradiated to kill the target microbes in a room. This research presents the development of a low-cost wireless UVC sensor that can be used to systematically track the UV irradiation dose on target surfaces during a UV disinfection procedure. We present key elements of the design of this device, which included a custom PCB, enclosure, operating software, and graphical user interface. The applicability of the system was assessed through an experiment where the devices were placed at 12 locations in a CT scan treatment room that was subject to a UVGI disinfection procedure using an autonomous UV robot. Over the course of three cleaning sessions, each lasting approximately 10 minutes, it was found that each site location received an average UVC dose of $13 \mathrm{~mJ} / \mathrm{cm}^{2}$, which is more than published D90 values for SARSCov-2, influenza, and a number of known pathogens that are commonly found in hospital settings. This study provides early validation of the potential effectiveness of low-power wireless UV level monitoring technology, which may form part of future distributed room sensing networks or as part of smart wearable devices carried by relevant hospital staff.
\end{abstract}

\section{INTRODUCTION}

Infection control procedures, including room cleaning and disinfection activities, play a critical role in hospitals to limit the spread of healthcare acquired infections (HCAIs) and reduce the pathways of transmission of infectious diseases, including SARS-CoV-2, MRSA, influenza, etc. Normally, room disinfection protocols involve the manual application of a chemical agent by a human worker. These approaches tend to be time and labour intensive, and their efficacy is subject to high variability due to the effects of human error. In recent years, alternative 'non-touch' disinfection methods such as ultraviolet germicidal irradiation (UVGI) have emerged, which can be administered rapidly and more uniformly within rooms.

UVGI is a light-based disinfection method that uses shortwavelength ultraviolet $\mathrm{C}$ (UVC) irradiation to inactivate microorganisms by destroying nucleic acids and disrupting their DNA, leaving them unable to perform vital cellular functions [1]. UVGI has many compelling advantages, including effectiveness against broad-spectrum organisms, lack of harmful residuals, reduced labour and consumable costs, and relative simplicity of operation within a healthcare environment.

\footnotetext{
*Funding for the project was provided for a grant from Science Foundation Ireland (SFI), as part of their COVID-19 Rapid Response Initiative (grant ID: 20/COV/0021).

${ }^{1}$ School of Engineering, Trinity College Dublin, Ireland

${ }^{2}$ Akara Robotics, Dublin, Ireland
}

There are three commonly encountered operational challenges associated with the deployment of UVGI systems. Firstly, parts of the room that do not get irradiated or that are occluded from irradiation may not receive the necessary UV dose during deployment. Second, since the intensity of the UV irradiance at a point is inversely proportional to the square of the distance to the UV source, the UV dose absorbed by surfaces close to the UVGI system is far greater than for surfaces further away. This non-linear relationship can make it challenging to optimally determine waypoints for mobile UVGI devices that ensure that required room coverage is achieved. Third, UVC light is a regulated technology that may cause harm if it's exposed directly to unprotected soft tissue (i.e., skin, eyes). Due to the potential for UVC irradiation to cause harm to humans, most UVGI systems require that rooms are evacuated of people prior to use. While the practice of evacuating rooms during the use of UVGI systems is common, it is conceivable that some lower-power UVGI systems may be used safely alongside people provided that their deployment meets legal UVC occupational safety requirements such as those specified in EU directive 2006/25/EC.

This paper addresses the need for new tools and test methods to inform the use of UVGI technologies in clinical settings. First, we introduce relevant prior work in the areas of UVGI, environmental UV sensing and robotics. We then present the design of a novel low-cost wireless UVC sensor alongside a description of a test method for obtaining estimates of UVC doses at different points in the room. Results from a real-world trial of the technology and the proposed test method are then presented and discussed.

\section{PRIOR WORK}

The majority of UVGI systems have tended to use either low-pressure (LP) mercury lamps or pulsed xenon arc technology as their UV source. LP mercury lamps are typically low-cost devices and come in a variety of different form factors and power ranges, and typically emit narrowband UVC light at a wavelength of $254 \mathrm{~nm}$. Two examples of LP mercury UVC lamp technology that has been deployed successfully in hospital settings are the Tru-D disinfection system [2], [3] and the Surfacide disinfection system [4]. Despite their low cost and popularity, LP mercury lamps have a limited operating lifetime, can create a hazard if the lamp is broken, and require several minutes to reach peak UV output. These limitations may be overcome using UV LED technology, which can come in a wider range of form factors, can reach their peak UVC outputs almost instantly, and can emit UVC at $265 \mathrm{~nm}$, the optimum wavelength for germicidal 
effectiveness [1]. While these features seem advantageous, their current high cost and low electrical efficiency relative to LP mercury lamps limit their applicability in many clinical use-cases [5]. Pulsed-xenon devices operate by generating extremely high-power pulsed UV output (approx. 120Hz) and emit irradiation across a spectrum of wavelengths with peaks in the UVA, UVB, and UVC ranges. The most widely used pulsed-xenon disinfection device is the Xenex LightStrike system, which is effective at reducing bioburden across a wide range of clinical settings [6], [7].

The amount of UVC energy required to kill microorganisms is well studied. A commonly cited statistic is the $D_{90}$ value, which describes the amount of UV energy (normally measured in $\mathrm{mJ} / \mathrm{cm}^{2}$ ) needed to bring about a $90 \%$ reduction in microbial content. Recent research indicates the the D90 value for SARS-CoV-2 is in the range of $1-5 \mathrm{~mJ} / \mathrm{cm}^{2}$ [8], [9], [10]. $D_{90}$ values for other microbes are commonly quoted in the literature and can be found in lookup tables, such as presented in [1] (p77-83).

Several studies have incorporated in-situ UVC measurements as a way to validate the likely effectiveness of UVGI treatment in a clinical or environmental setting. Lindblad et al. used UVC sensitive cards along with a digital UVC meter to measure the UV dose on target surfaces in a room during a UVGI treatment procedure; the recorded values were then cross referenced with the ClorDiSys Ultraviolet Light Disinfection Data Sheet [11]. A similar approach was followed by Masse et al. when comparing the performance of four different UVGI platforms [12]. Lindsey et al. used a digital UV sensor to measure the UV irradiance at 49 locations in an ambulance compartment [13]. While each of these studies benefited from UVC sensing, the process of acquiring the data required considerable manual effort (i.e. the placing of disposable UV sensitive cards, manual setup of a UV sensor module, manual logging of the data) and were generally not readily scalable within day-to-day clinical workflow.

\section{Methods}

The likelihood of a microorganism being inactivated by UVGI is dependent on the UVC exposure dose $(D$, $\mathrm{mJ} / \mathrm{cm}^{2}$ ), which can be expressed as the integral of the UVC irradiance $\left(I_{R}, \mathrm{~mW} / \mathrm{cm}^{2}\right)$ with respect to time.

$$
D=\int_{0}^{t} I_{R} d t
$$

In the following subsection, we describe the design of a bespoke UVC sensor module. This module used a commercially available UVC sensor to read the instantaneous UV irradiance and an on-board microprocessor to determine the UVC dose over a specified period.

\section{A. Wireless UVC Sensor module}

A prototype UVC sensor module was first designed. The module utilized an off-the-shelf UVC sensor (GUVCT21GH, Genicom Co., Ltd), rated for detecting up to $5 \mathrm{~mW} / \mathrm{cm}^{2}$ of UVC irradiation with a spectral range

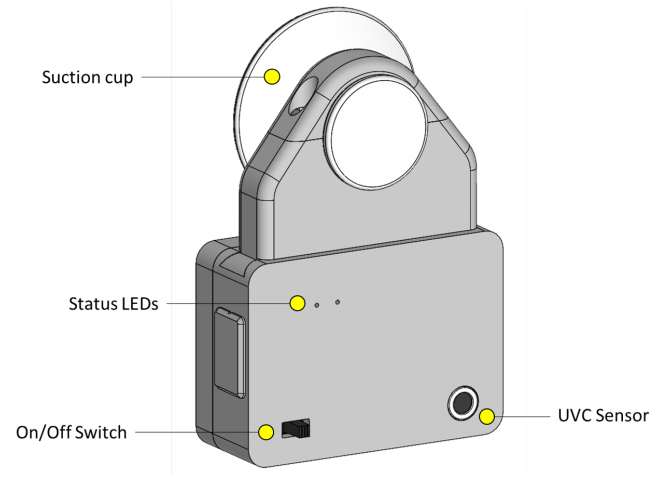

(a)

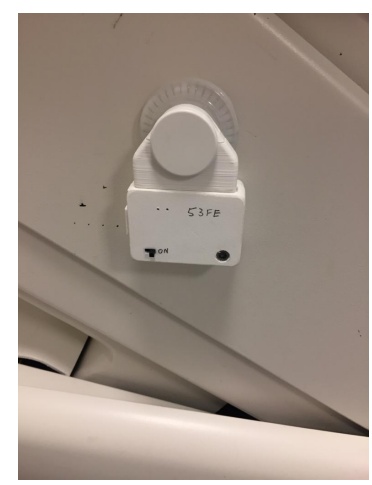

(b)

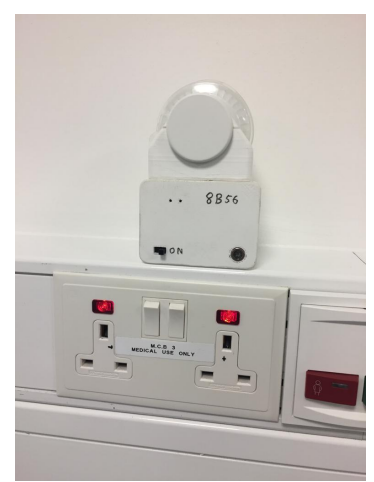

(c)
Fig. 1. The wireless UVC sensor used in this study. (a) Labelled schematic of the senor module, $(b, c)$ Photos of the UVC sensor device mounted to surfaces in a clinical setting during testing.

of $220 \mathrm{~nm}-280 \mathrm{~nm}$. The analogue signal from the sensors was measured by a 16 bit analogue to digital converter (ADS1115, Texas Instruments). Incoming sensor readings were analysed using an ESP32 microcontroller (Espressif systems); this module was chosen for its processing capability, low-cost, and built-in wifi/Bluetooth connectivity. The electronics were housed within a 3D-printed enclosure that allowed for the modular addition of a suction cup, thus enabling it to be firmly positioned on vertical surfaces such as walls and cupboards. A $1000 \mathrm{mAh}$ lithium polymer battery was mounted within the enclosure and provided in excess of $30 \mathrm{hrs}$ battery life. Images of the wireless sensor module are given in Fig. 1 .

Each device communicated their readings using a Bluetooth low energy (BLE) data packet that advertised to other BLE devices in their vicinity. The BLE data packet contained the following information:

- Current UVC fluence $\left(\mathrm{mW} / \mathrm{cm}^{2}\right)$.

- Total UVC light energy/unit area $\left(\mathrm{mJ} / \mathrm{cm}^{2}\right)$.

- Time over which the light energy was measured.

- Current battery level.

- Unique MAC address of the device.

A graphical user interface (GUI) that could communicate with the UVC sensors was developed using MatLab (Mathworks) and run as an executable on a host laptop running Windows 10. Since the sensors used an advertisement packet 
to send the data, the BLE receiver was able to read from multiple sensors simultaneously without the need to connect to each one individually. A screenshot of the GUI is given in Fig. 2 .

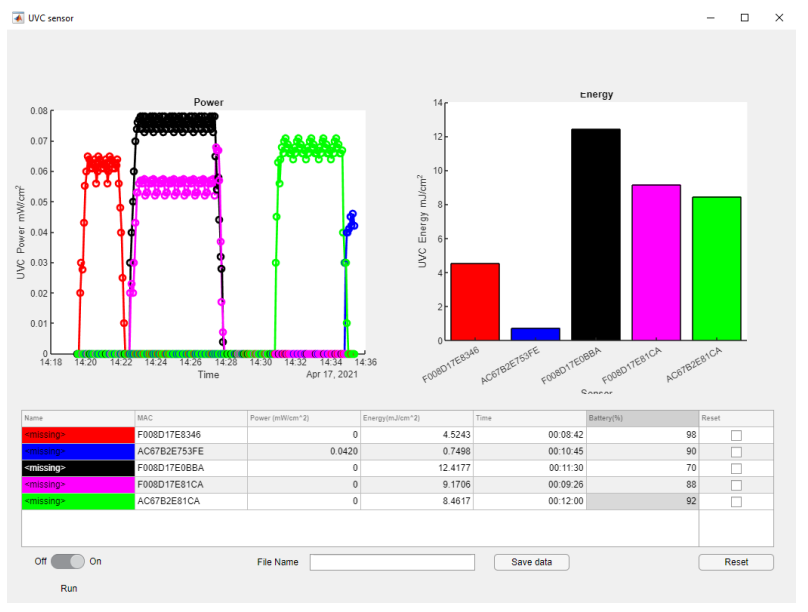

Fig. 2. Screengrab of the graphical user interface used to visualise the sensor readings. The table at the bottom of the screen lists the names and colors of the UVC devices on the network. The box in the upper left of the screen shows the instantaneous UVC irradiance of each registered sensor. The box in the upper right shows the UVC dose over a reference time period, which can be set through the software program.

\section{B. Experimental Procedure}

An in-hospital trial was conducted to explore the utility of the UV sensor module. The trial took place in a CT scan treatment room where a robotic UVGI system had been implemented and was regularly being used to disinfect surfaces in the room. The UVGI device used in this work was the UVC robot developed by Akara (Fig. 3(a)). This platform possessed three LP mercury lamps (TUV 36W SLV, Philips) that were housed in a vertical column that could be rotated to expose the robot's lamps to target surfaces in the room.

The robot was programmed to autonomously move to a series of 21 waypoints throughout the room. The robot waited for 15 seconds at each waypoint before moving to the next one. The approximate location of these waypoints, and the forward-facing direction of the UV column at each location is given in Figure 3(b) Excluding the 3-4 minute warm-up period for the UV lamps to reach a steady state value, the total time of each robot test run was approximately 9 minutes 30 seconds.

Using the UVC sensors, the UVC dose at 12 locations in the room was measured (Fig. 3(b)). The sample locations included high-touch surfaces and/or areas where human activity was common. Eight of the surfaces sampled were parallel to the robot's UV lamps, while the remaining 4 were perpendicular. The height of the sampled surfaces also varied; low surfaces included foot pedals (Locations 09 and 11), while high surfaces included glove holders (Locations 04 and 08) and a high press (Location 06).

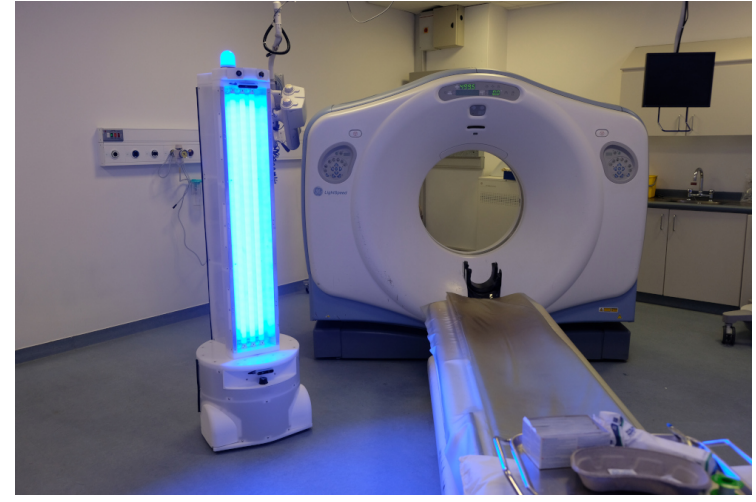

(a)

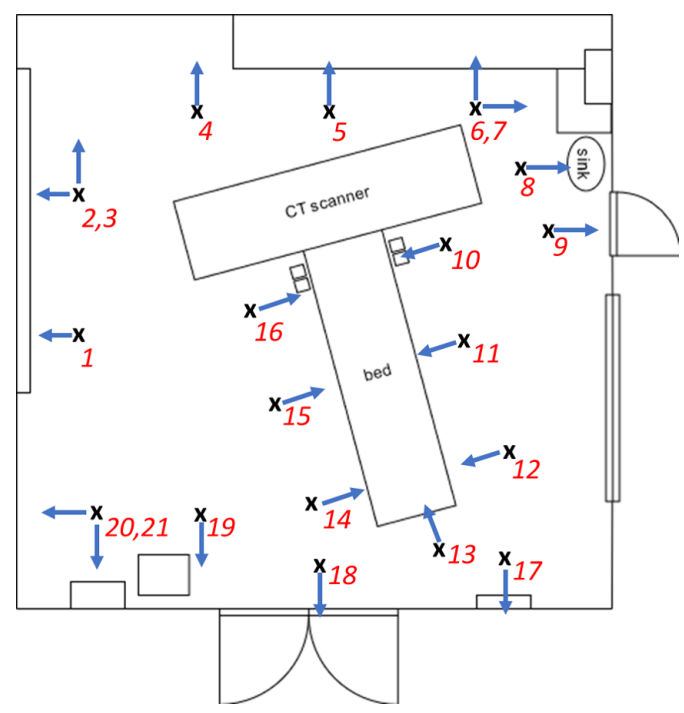

(b)

Fig. 3. Details of the robotic UVGI procedure: (a) photo of the Akara UV robot, (b) diagram showing each of the waypoints and forward-facing direction of the UV lamp (indicated by the arrow).

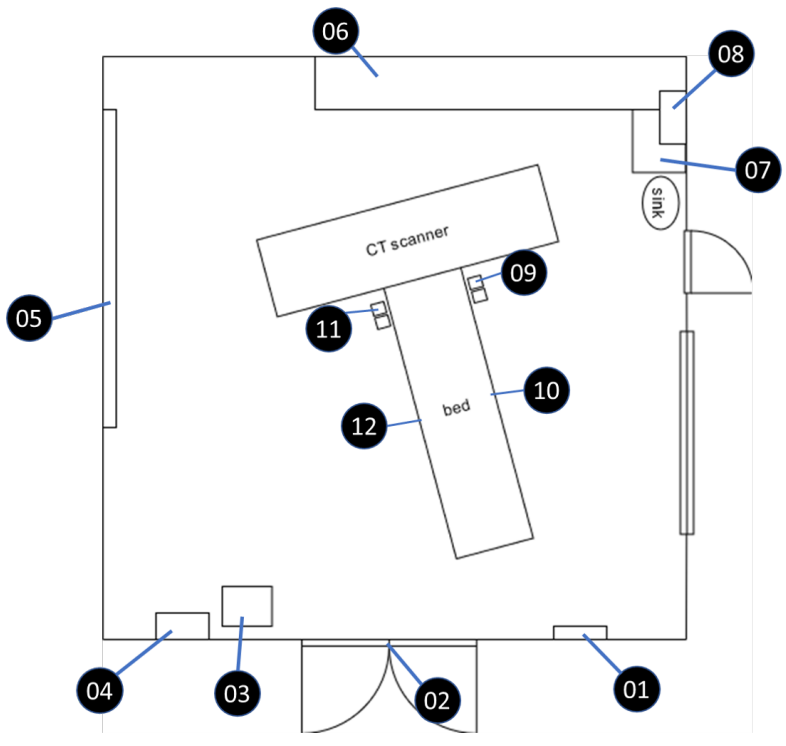

Fig. 4. Photo of the Akara robot irradiating surfaces in a CT room. 


\section{RESUlTS}

Three UVC readings were taken at each location during UVGI treatment. To ensure the measurements were robust to minor changes in the location of the sensor, the modules were re-positioned to another location in the general vicinity of the target surface after each test. Since only 5 UVC sensors had been produced at the time of the study, a total of eight robotic UVGI cleaning procedures were performed to collect the data. A barchart showing the recorded UVC dose at each location is given in Fig. 5. The mean UVC dose and associated standard deviation at each location is presented in Table []

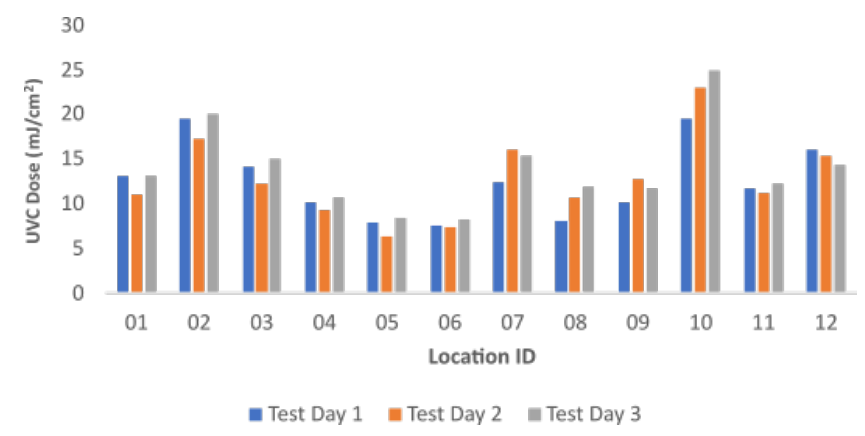

Fig. 5. Graph of the recorded UVC dose at each of the 12 locations over over the course of a UVGI disinfection procedure using the Akara robot.

TABLE I

RECORDED UVC DOSE AT EACH OF THE 12 LOCATIONS OVER OVER THE COURSE OF A UVGI DISINFECTION PROCEDURE USING THE AKARA ROвот.

\begin{tabular}{|c|c|c|c|c|c|}
\hline Location & Test Day 1 & Test Day 2 & Test Day 3 & Average & Std Dev \\
\hline 01 & 13.17 & 11.00 & 13.04 & 12.40 & 0.99 \\
\hline 02 & 19.45 & 17.34 & 19.97 & 18.92 & 1.14 \\
\hline 03 & 14.15 & 12.22 & 15.08 & 13.82 & 1.19 \\
\hline 04 & 10.16 & 9.32 & 10.63 & 10.03 & 0.54 \\
\hline 05 & 7.82 & 6.30 & 8.39 & 7.50 & 0.88 \\
\hline 06 & 7.58 & 7.39 & 8.32 & 7.77 & 0.40 \\
\hline 07 & 12.47 & 15.98 & 15.29 & 14.58 & 1.52 \\
\hline 08 & 8.00 & 10.62 & 11.92 & 10.18 & 1.63 \\
\hline 09 & 10.07 & 12.69 & 11.67 & 11.47 & 1.08 \\
\hline 10 & 19.48 & 23.04 & 24.97 & 22.50 & 2.27 \\
\hline 11 & 11.78 & 11.21 & 12.15 & 11.71 & 0.39 \\
\hline 12 & 16.04 & 15.30 & 14.38 & 15.24 & 0.68 \\
\hline Average & 12.51 & 12.70 & 13.81 & & \\
\hline Std. Dev & 3.98 & 4.43 & 4.54 & & \\
\hline
\end{tabular}

\section{DISCUSSION}

The mean UVC dose measured at each location was $13.01 \pm 4.36 \mathrm{~mJ} / \mathrm{cm}^{2}$. This exceeds the D90 value for SARSCoV-2, estimated to be in the region of $1-5 \mathrm{~mJ} / \mathrm{cm}^{2}$ [8], [9], [10]), as well as other common pathogens including E. coli $\left(3.5 \mathrm{~mJ} / \mathrm{cm}^{2}\right), C$. difficile $\left(6 \mathrm{~mJ} / \mathrm{cm}^{2}\right), S$. aureus $\left(2.6 \mathrm{~mJ} / \mathrm{cm}^{2}\right)$, MRSA $\left(3.2 \mathrm{~mJ} / \mathrm{cm}^{2}\right)$, among others [14]. Only 2 of the 12 locations experienced a dose less than $10 \mathrm{~mJ} / \mathrm{cm}^{2}$ (locations 05 and 06). These results provide meaningful insights which may be used to further shorten the overall irradiation period (i.e., by reducing time the UV robot spends at locations with large UVC doses), or to extend the period spent at certain waypoints to ensure that minimum UVC doses are achieved on target surfaces.

The variance over the three UVC measurements fluctuated at each location. The location with the highest standard deviation (proportional to the magnitude of the UVC reading at that point) was at location 08 (16\% of mean UVC dose), while the lowest occurred at location 11 (3\% of mean UVC dose). These variances can be likely be attributed to small differences in the positioning of the robot at each waypoint, rather than noise in the sensor itself. Since the robot moved between waypoints autonomously using only its on-board sensors, localization errors are likely to have led to small deviations in the end position. Since the UVC irradiance at the surface is inversely proportional to the square of the distance to the UVC source, even quite small changes in distance can have a significant effect on the UVC fluence received at the surface.

The UVC values recorded at these locations may be conservative since the sensitivity cone for the UVC sensors was only $60^{\circ}$. Given the $1.2 \mathrm{~m}$ span of the UVC lamps used on the robot, it is possible that the positioning of the UVC source relative to the sensor may have led to some UVC irradiance not being recorded. Future versions of the sensor module may account for this by including more than one UVC sensor or choosing a different UVC sensor with a larger sensitivity cone.

\section{CONCLUSIONS}

Through a pilot study that involved the deployment of a UVC robot in a hospital setting, we demonstrated the feasibility of using multiple UVC sensor modules to generate a quantitative map of the UVC irradiance in a room during a UVC procedure. The resulting data produced by the sensors enabled verification that surfaces had been irradiated with a UVC dose in excess of the D90 value of numerous common pathogens, including SARS-CoV-2. Further analysis of the data may prove useful in optimizing the robot's trajectory, providing a systematic way to ensure minimum threshold UVC doses are achieved on all target surfaces.

Although it was beyond the scope of this study, we observed that only the UVC sensors adjacent to the robot registered readings during the UVGI treatment. This suggests that reflections and background radiation was otherwise low. Based on this observation, it's conceivable that a person may have been able to be present in the room, while staying within occupational safety requirements for UVC technology. Using the UVC sensor modules to investigate the feasibility of human-robot co-location during UVGI remains interesting future work.

\section{ACKNOWLEDGEMENTS}

We are grateful for support from the Midlands Regional Hospital Tullamore and the Health Service Executive (HSE) for supporting the clinical testing performed in this research. 


\section{CONTRIBUtions}

All work pertaining to the design and manufacture of the UVC sensor modules was undertaken by employees of Akara Robotics. All work concerning the testing and evaluation of the sensors was undertaken by researchers at Trinity College.

\section{REFERENCES}

[1] W. Kowalski, Ultraviolet germicidal irradiation handbook: UVGI for air and surface disinfection. Springer science \& business media, 2010.

[2] M. M. Nerandzic, J. L. Cadnum, M. J. Pultz, and C. J. Donskey, "Evaluation of an automated ultraviolet radiation device for decontamination of clostridium difficile and other healthcare-associated pathogens in hospital rooms," BMC infectious diseases, vol. 10, no. 1, pp. 1-8, 2010.

[3] W. A. Rutala, M. F. Gergen, B. M. Tande, D. J. Weber, et al., "Rapid hospital room decontamination using ultraviolet (uv) light with a nanostructured uv-reflective wall coating," Infect Control Hosp Epidemiol, vol. 34, no. 5, pp. 527-529, 2013.

[4] K. Bedell, A. H. Buchaklian, and S. Perlman, "Efficacy of an automated multiple emitter whole-room ultraviolet-c disinfection system against coronaviruses mhv and mers-cov," infection control \& hospital epidemiology, vol. 37, no. 5, pp. 598-599, 2016.

[5] S. E. Beck, H. Ryu, L. A. Boczek, J. L. Cashdollar, K. M. Jeanis, J. S. Rosenblum, O. R. Lawal, and K. G. Linden, "Evaluating uv-c led disinfection performance and investigating potential dual-wavelength synergy," Water research, vol. 109, pp. 207-216, 2017.

[6] M. M. Nerandzic, P. Thota, T. Sankar, A. Jencson, J. L. Cadnum, A. J. Ray, R. A. Salata, R. R. Watkins, and C. J. Donskey, "Evaluation of a pulsed xenon ultraviolet disinfection system for reduction of healthcare-associated pathogens in hospital rooms," infection control \& hospital epidemiology, vol. 36, no. 2, pp. 192-197, 2015.

[7] B. Casini, B. Tuvo, M. L. Cristina, A. M. Spagnolo, M. Totaro, A. Baggiani, and G. P. Privitera, "Evaluation of an ultraviolet c (uvc) light-emitting device for disinfection of high touch surfaces in hospital critical areas," International journal of environmental research and public health, vol. 16, no. 19, p. 3572, 2019.

[8] J.-L. Sagripanti and C. D. Lytle, "Estimated inactivation of coronaviruses by solar radiation with special reference to covid-19," Photochemistry and photobiology, vol. 96, no. 4, pp. 731-737, 2020.

[9] B. Pendyala, A. Patras, B. Pokharel, and D. D'Souza, "Genomic modeling as an approach to identify surrogates for use in experimental validation of sars-cov-2 and hunov inactivation by uv-c treatment," Frontiers in Microbiology, vol. 11, 2020.

[10] M. Heßling, K. Hönes, P. Vatter, and C. Lingenfelder, "Ultraviolet irradiation doses for coronavirus inactivation-review and analysis of coronavirus photoinactivation studies," GMS hygiene and infection control, vol. 15, 2020.

[11] M. Lindblad, E. Tano, C. Lindahl, and F. Huss, "Ultraviolet-c decontamination of a hospital room: Amount of uv light needed," Burns, vol. 46, no. 4, pp. 842-849, 2020.

[12] V. Masse, M. J. Hartley, M. B. Edmond, and D. J. Diekema, "Comparing and optimizing ultraviolet germicidal irradiation systems use for patient room terminal disinfection: an exploratory study using radiometry and commercial test cards," Antimicrobial Resistance \& Infection Control, vol. 7, no. 1, pp. 1-7, 2018.

[13] W. G. Lindsley, T. L. McClelland, D. T. Neu, S. B. Martin Jr, K. R. Mead, R. E. Thewlis, and J. D. Noti, "Ambulance disinfection using ultraviolet germicidal irradiation (uvgi): Effects of fixture location and surface reflectivity," Journal of occupational and environmental hygiene, vol. 15, no. 1, pp. 1-12, 2018.

[14] ClorDiSys, Ultraviolet Light Disinfection Data Sheet. Rev. 12-2020. 